\title{
Modeling the Structures of Oriented Macrofiber Polymer Composites as Capillary-Porous Bodies
}

\author{
Aleksandr E. Kolosov (D), Aleksandr V. Gondlyakh, Volodymyr I. Sivetskii, \\ Elena P. Kolosova, Volodymyr V. Vanin, Dmytro E. Sidorov, Aleksandr L. Sokolskiy, \\ Valeriy Yu Shcherbina, and Igor I. Ivitskiy
}

National Technical University of Ukraine, Igor Sikorsky Kyiv Polytechnic Institute, 37 Prospect Peremohy, Kyiv 03056, Ukraine

Correspondence should be addressed to Aleksandr E. Kolosov; a-kolosov@ukr.net

Received 9 June 2020; Accepted 18 July 2020; Published 24 August 2020

Academic Editor: Barbara Liguori

Copyright ( 2020 Aleksandr E. Kolosov et al. This is an open access article distributed under the Creative Commons Attribution License, which permits unrestricted use, distribution, and reproduction in any medium, provided the original work is properly cited.

\begin{abstract}
The problems of modeling the structure and design of technical parameters for forming of oriented macrofiber polymer composites are analyzed. The considered approaches are based on the application of geometric modeling of the structure of oriented fibrous fillers and the design of the parameters of technical means for forming oriented macrofiber polymer composite materials. Peculiarities of the application of the phenomenological and structural approaches to the design of structural and technological parameters of technical means and to the calculation of the elements of composite structures based on oriented macrofibrous fillers are considered. The technique for determining the structural characteristics of oriented macrofibrillar fillers based on its adequate geometric model as capillary-porous bodies is given. As the structural characteristics, the porosity, the specific internal surface, and also the effective (hydraulic) capillary radius of oriented macrofibrous fillers are considered. It is noted that the obtained structural models are used, in particular, to the prognosis of the design and technological parameters of the technical means for molding macro-fiber polymer composite materials.
\end{abstract}

\section{Introduction}

Currently, thermosetting polymer composite materials (PCMs) are one of the most popular structural materials in many innovative industries $[1,2]$. So, for example, the increasing use of such PCMs in the aircraft industry [3-5], for critical structural elements of rocket and space technology $[6,7]$, in shipbuilding, for example, for the repair of ship superstructures [8], in manufacturing protective ammunition (body armor, helmets, etc.), for military personnel [9], in various applications in biomedical engineering [10], in forming the paddles of wind power plants, including fiberglass [11], and in many other areas of science and technology should be noted.

Moreover, every year the scope of PCMs is expanding, and production volumes are increasing. There is a wide variety of thermoset PCMs types that differ in their properties. This allows you to select the optimal composite material for specific operating conditions [12-14]. No less important from a practical point of view, cost analysis in the formation of thermoset composites [15] allows you to choose the most economical method of molding PCMs.

To date, considerable experience has been accumulated in the world in the field of modeling the structure and design parameters of technical means for molding reactoplastic composite materials (PCMs) for structural purposes [16-18]. This class of materials successfully competes and displaces many traditional materials in the most diverse fields of modern technology [19]. The effectiveness of using laminated structures to create high-strength multilayer structural PCMs should be emphasized [20-23], including prediction of their evolutionary stratification during operation activity, based on adequate structural models of laminated capillaryporous structures [24].

Filled PCMs are characterized by significant heterogeneity, in particular, the presence of an interphase layer. 
Therefore, the development of new PCMs and the prediction of their operational properties, along with the design of the parameters of technical means for their molding, is one of the important tasks of modern chemical engineering.

At present, there are several approaches to solving the problem of predicting the operational properties of PCMs. All of them differ in the degree of generality and have a number of shortcomings. So, most of the models do not take into account the structure of the material, which is one of the main factors that determines its properties.

A number of models are limited by the number of system components. In this case, the calculated values are purely evaluative, giving, as a result, a large "scatter" of acceptable values. All this testifies to the urgency of the problem of creating new methods for modeling the structure and operational properties of the PCMs.

\subsection{Analysis of the Literary Sources and the Formulation of the} Problem. It should be noted that considerable progress has been made in the design of the technology for molding macrofiber reactoplastic PCMs using the appropriate structural models [25-28]. At the same time, the modeling of the PCM structure is an exceptionally complex scientific and technical problem for a number of reasons related to the peculiarities of their structural organization. It is especially important to establish the relationship between the imperfections in the structural organization of the designed PCMs, which leads to the formation of defects both during production and during PCMs operation activity [29, 30].

Moreover, there are still quite clear theoretical representations, including mathematical dependencies and corresponding methods, allowing to predict with sufficient accuracy the geometric parameters of adequate structural models of such materials $[27,28]$. Such models are used, in particular, to predict the design and technological parameters of the technical means for molding PCMs [12], for example, the parameters of the "wet" winding process of oriented fibrous fillers preimpregnated with a polymer binder onto a forming mandrel [31]. In connection with this, when designing such materials, a whole range of modeling methods is used to reduce material and time costs; among them are the mathematical, experimental-statistical, and computer.

It is important to adhere to a systematic approach at all stages of design and subsequent manufacturing of structural materials, the adaptation of which for structurally parametric modeling of oriented reinforced PCMs as capillaryporous bodies is presented in the study [32]. For example, the parameters of radiation heating of viscous polymer matrices during the molding of composite products from the perspective of a systematic approach and taking into account the structural features of the impregnated fibrous fillers were considered in work [33]. Examples of the use of the technique of structural and parametric modeling for the design of technical facilities on the example of ultrasonic treatment when impregnating fibrous fillers as capillary-porous bodies can be found in studies [34-36]. Different technological aspects of physicochemical dynamics of structure formation and rheology during production of PCMs with using the same approach in modeling are analyzed in [37].

The main structural elements of PCMs are the polymer matrix, which performs a consolidating role, and the reinforcing filler, which perceives the main load in the power composite [7]. At the same time, one of the main roles belongs to the above modeling methods for geometric modeling, which works in a "bundle" with mathematical modeling. Moreover, when manufacturing fibrous PCMs, various schemes of fiber packing are used, depending on the functional purpose of the composites.

An important class of PCMs are spatially reinforced PCMs based on oriented (single directed) (macro) fibrous fillers (OFFs) [7]. One of the most common schemes for laying fibers in the formation of spatially reinforced PCMs is the $4 \mathrm{D}$ scheme [38], in accordance with which the reinforcing fibers are arranged along four diagonals of the cube (see Figure 1).

The geometry of the spatial reinforcement is created, proceeding primarily from the conditions of the PCM load, and is intended to provide a targeted anisotropy of its properties [38]. At the same time, this $4 \mathrm{D}$ scheme makes it possible to obtain a balanced structure of the reinforced composite. Taking into account the symmetry of the structure, it should be noted that the elastic properties of the PCM obtained on the basis of the 4D scheme also have symmetry elements. In this case, one of the reinforcement directions is the third-order elastic symmetry axis.

When the coordinate system, which is connected by one axis with the direction of the fibers, is rotated through an angle of $120^{\circ}$ in the plane of the tetrahedron base, all the elastic properties of such a composite remain due to symmetry. Therefore, such a reinforcement structure is more promising for increasing the stiffness of the composite, for example, during shear, in comparison with other reinforcement schemes [38]. Geometric schemes of spatially reinforced with macro-fibers structures with different reinforcement structures are presented in the handbook [7].

The development and implementation of spatially reinforced materials are based on the development and practical implementation of relevant theories. Moreover, these theories have distinctive features for modeling the structure, properties, and technical means of molding PCMs based on macro- and microfillers. At the same time, these theories are the basis for the development of technologies and technological devices that implement them [1].

Modeling the structures of oriented macro-fiber polymer composites as capillary-porous bodies is one of the approaches developed by the authors to the structural-parametric modeling of such PCMs. This is due to the fact that reinforcing fibrous fillers during capillary impregnation with liquid polymer binders are actually capillary-porous bodies; that is, the interfiber space (spatial framework) of the composite is a pore structure. And after impregnation of the OFFs, the stage of their curing follows, which "fixes" the final structure of the fibrous composite.

Capillary models are a pores space in the form of a system of channels with certain geometric properties. Some (most often used) structural-network models of capillary 


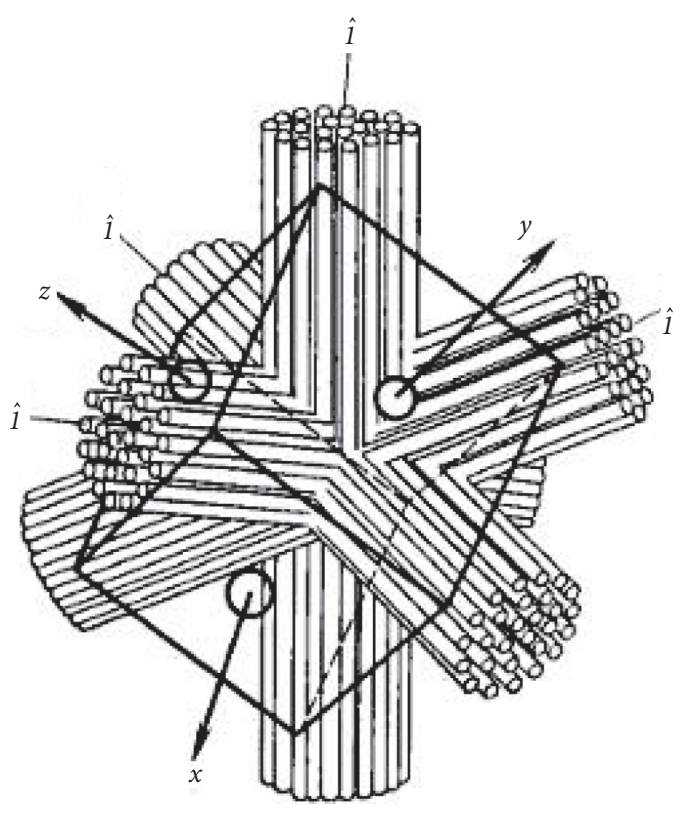

FIGURE 1: Four-directional reinforcement scheme and coordinate system location; $x, y, z$ are the principal axes of elastic symmetry; $\hat{\imath}$ is the direction of the reinforcement [38].

bodies in the form of one-dimensional models are shown in Figure 2 [39].

In the model of identical direct channels (Figure 2(a)), the main parameters of the model are the volume fraction of the channels and their diameter. In the model of identical parallel meandering channels (Figure 2(b)), an additional parameter of the curvature of the capillaries is introduced, the tortuosity coefficient $\beta_{0}$ [40].

To take into account stagnant (dead-end) zones (analog of the "bottleneck" effect) in porous materials and their influence on the impurity dispersion, a channel model with dead-end branch pores is used (Figure 2(c)). And in order to take into account the effect of dead-end pores, an additional quantity is introduced, the volume fraction of the dead-end pores.

In cases where fluid filtration is modeled by a nonuniform problem and additional fluid transport occurs across channels, a model of channels connected with each other by a developed system of micropores is applied (Figure 2(d)). This idealization was called the model of parallel capillaries with an ideal connection. In the serial models (Figure 2(d)), the variability of the pore cross section is taken into account, i.e., the presence of contractions and extensions along the length. The diameter of the capillary in the serial models varies abruptly. Corrugated capillaries (Figure 2(e)), in contrast to the serial models, have a diameter continuously varying in length.

Replacing the pore space with a system of one-dimensional channels, in general, one can take into account the tortuosity, corrugation of pores, their various sizes and shapes, and the presence of stagnant zones. But such important properties of porous bodies, as the interrelation of individual capillaries and the intersection of pore space, are practically not taken into account. Therefore, a system of one-dimensional capillaries can be used to adequately describe capillary phenomena only for idealized cases. It is believed that lattice models most fully reflect the spatial structure of porous media, as well as the interconnection of their constituent elements.

Some models of regular (quasi-regular) lattices are shown in Figure 3. The model of a square lattice, in which the particles of different shapes are located (Figure 3(a)), can be used, for example, to describe the processes of impregnation and filtration of liquid media in porous media formed by dispersed particulates. To describe similar processes in porous media consisting of particles, bidisperse models of porous media are used (Figure 3(b)).

Such models are applicable, for example, to describe the processes taking place in the catalyst grains (microporous bodies in a granular layer). Another example of the use of such models is the description of the extraction process of plant tissues characterized by the regular structure of micro- and macropores. When manufacturing PCMs based on cross-reinforced OFFs, for example, glass of textolite, different types of stitched satin, and twill and plain weave are used as fiberglass reinforcing fillers [42] (see Figure 4).

The structure of the interlacing of such tissues can be represented as a linear model (in the case of impregnation of only one layer of tissue, which is most often the case in practice) and a regular lattice model (in the case of simultaneous impregnation of several layers of fabric or glass mats located one above the other).

It should also be noted that symmetry is characteristic for the structure of such reinforcing fabrics (see Figure 4), as well as for the structure of spatially reinforced composites (see Figure 1).

It is known that two-dimensional (2D) laminated (fabric) composites (see Figure 4) are characterized by high planar rigidity and strength [43]. At the same time, there was a need for composite materials with improved out-of-plane properties, that is, in thickness. This necessitates the replacement of $2 \mathrm{D}$ laminated composites with three-dimensional (3D) textile structures. In this case, the resistance to delamination due to the presence of the $\mathrm{z}$-binder increases the impact strength and resistance to damage of such systems of materials according to [44-46].

Generally, 3D fabric-based composites can be divided into two main groups depending on how deeply the polymer binder penetrates through the fabric (transverse capillary impregnation). When the polymer binder penetrates the entire thickness of the fabric, it is called through-thickness (TT) blocking (see Figures 5(a) and 5(c)). And if the binder holds only adjacent layers (see Figure 5(b)); then it is classified as interlayer (LTL).

The above classification is further can be divided on the categories according to the interlacing angle of the structure. The first category is the angle interlock (AI) in which the interlacing angle between the binder and weft yarns can have any value except $90^{\circ}$ (Figure $5(\mathrm{c})$ ). The second category is a special case of the first one. The orthogonal interlock (ORT) (Figure 5(a)) occurs when the interlacing angle between the binder and weft yarns is equal to $90^{\circ}$ [43]. 


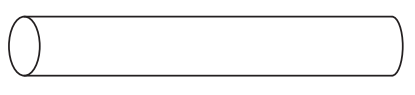

(a)

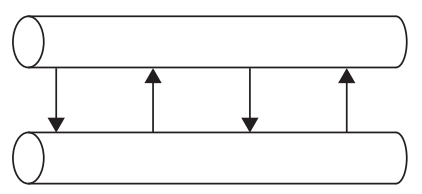

(d)

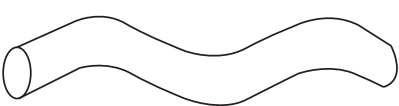

(b)

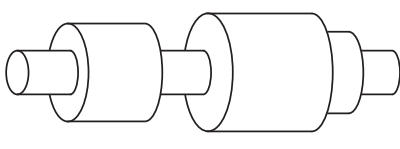

(e)

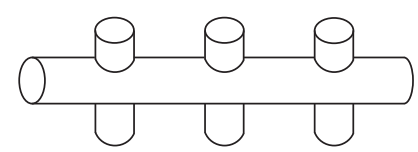

(c)

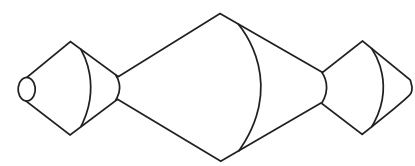

(f)

FIGURE 2: The simplest capillary models of porous bodies [39]: (a) a straight channel; (b) sinuous channel; (c) a channel with departing deadlock pores; (d) a system of parallel channels with an ideal connection; (e) serial model; (f) periodic corrugated channel.
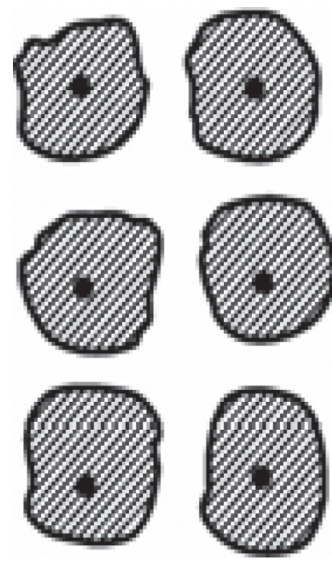

(a)

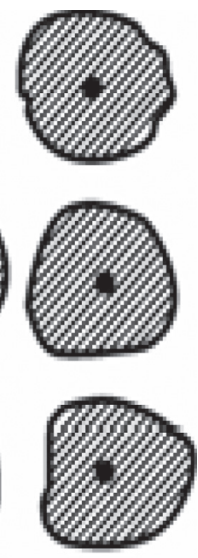

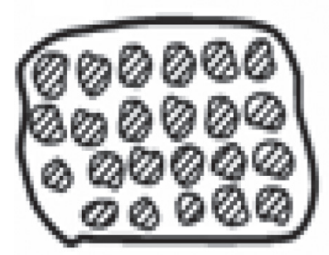
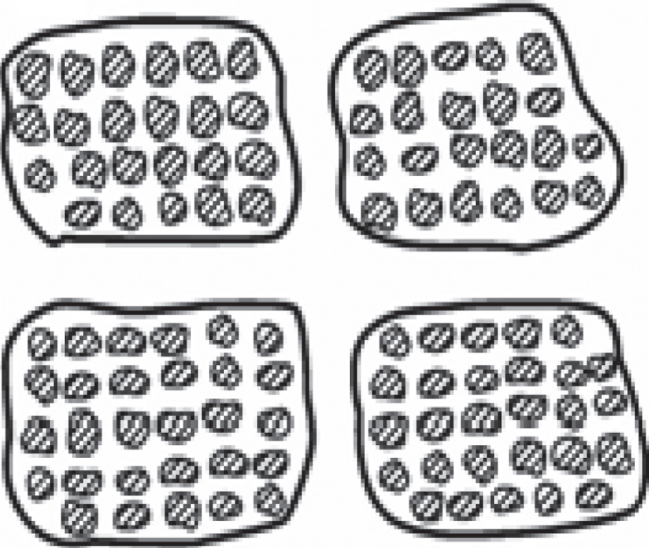

(b)

Figure 3: Lattice regular models of porous media [39, 41]: (a) the system of particles at the lattice sites; (b)-bidisperse model.

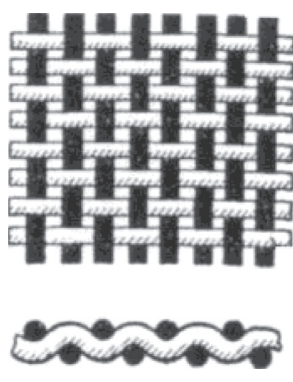

(a)

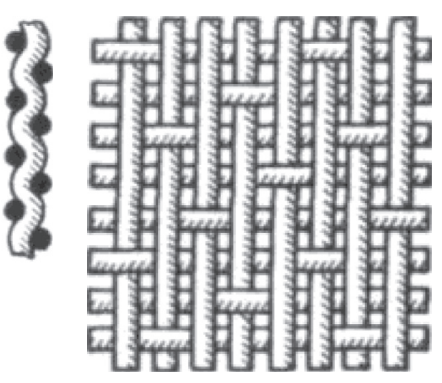

(b)

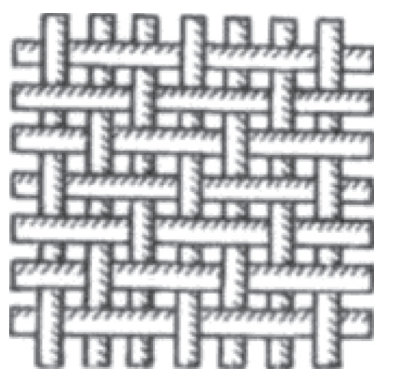

(c)

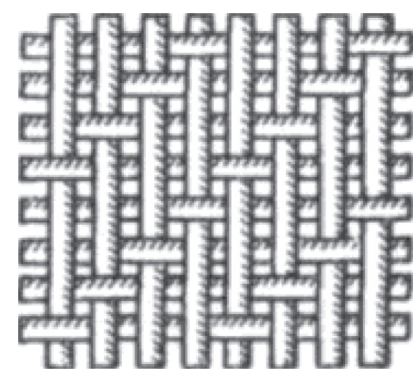

(d)

FIgURE 4: Some types of interlacing of glass fabrics [42]: (a)-linen; (b)-satin; (c) and (d)-twill (fabrics of type 1/2 and 3/1).

The weave pattern used during the weaving process can also affect the classification of 3D woven composites. For example, in the case of ORT weave, the frequency of the $z$ binder sweeping the top and bottom surfaces of the weave can vary from plain (Figure 6(a)) to twill (Figure 6(b)) or satin (Figure 6(c)) pattern. This will directly affect the unitcell size, degree of crimp, elastic response, and damage/ delamination resistance as highlighted previously in [46-48].
The applications of 3D woven composites have been growing tremendously in the industry recently [49-51]. In applications that require load transfer around a bend such as curved beams, T-joints, and brackets, $3 \mathrm{D}$ woven $\mathrm{AI}$ and LTL architectures have been utilized [52]. The main advantages observed for these architectures are that they have better interlaminar shear and radial stress resistance.

In addition, in study [44], successfully using 3D woven AI and LTL architectures in truss beams with integrated 


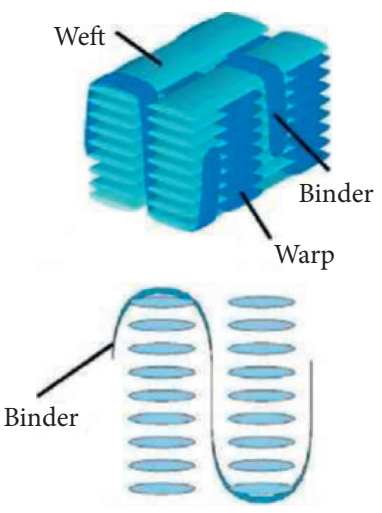

(a)

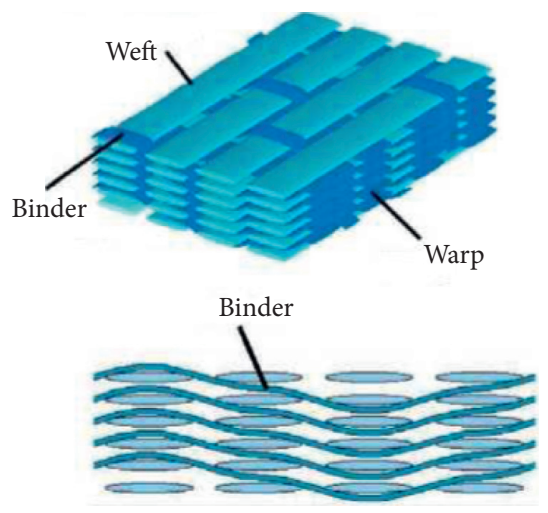

(b)

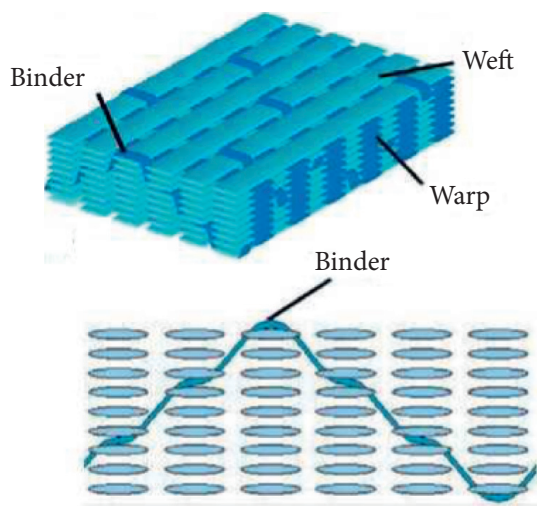

(c)

Figure 5: Types of 3D woven composites based on the binder path: ORT (a), LTL (b) and AI (c) [46].

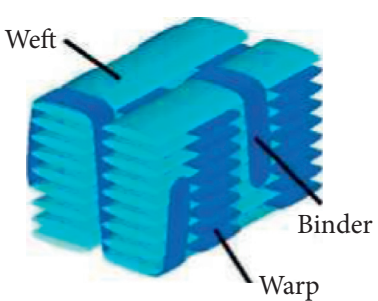

(a)

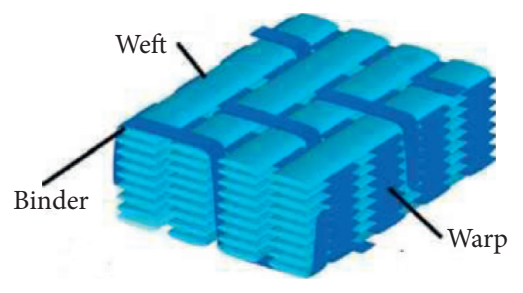

(b)

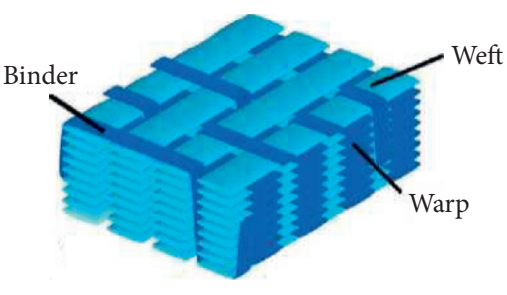

(c)

FIgURE 6: Examples of possible weave binding patterns for ORT: plain (a), twill (b) and satin (c) [46].

off-axis stiffeners was reported. For example, 3D woven LTL composites have been utilized in the automotive industry [53-55]. The use of ORT woven composite in ultralight weight heat exchangers [56] and for manufacturing engine casings and fan blades for A-320-neo, 737-MAX, and Comac C-919 is known [57].

In the general case, the concepts of capillary-porous media are much broader than those described above. Moreover, the more complex the structural model of such bodies, the more complex its geometric model and mathematical modeling of the processes of transport of a liquid medium in it. Therefore, it is necessary to find a certain balance when choosing an adequate geometric (structural) model of capillary-porous bodies for a particular technological process.

In this paper we present an analysis of only a few developed approaches to solving this problem. Such approaches are based on the application of geometric and mathematical modeling of the structure of OFFs and the design of technical parameters for shaping oriented macrofiber PCMs, in particular, the basic technological processes of capillary impregnation.

1.2. Purpose and Objectives of the Study. The aim of this study is adaptation of modeling the structures of oriented macrofiber polymer composites for developing of methodological approach for the geometric and computer models of the basic technological process of "free" impregnation of oriented fibrous fillers as capillary-porous bodies with liquid polymeric binders.
To achieve this goal, the following tasks are formulated.

(1) An analysis of approaches to modeling the structure of the cross section of thin section of oriented fibrous composites, including the construction of histograms of the distribution of distances between adjacent fibers in the cross section of thin section.

(2) Microstructural analysis of the cross section of thin section of oriented fibrous composites, including the construction of histograms of the distribution of distances between adjacent fibers in the cross section of thin section.

(3) Obtaining analytical dependencies of the curve distributions of distances between adjacent fibers in a cross section of thin section and modeling on their basis the structural parameters of oriented fibrous fillers as capillary-porous bodies, namely, porosity, effective capillary radius, and specific surface area.

(4) Experimental determination of the technological characteristics of liquid epoxy oligomers, namely, the wetting angle, wetting ability, the maximum height of impregnation, and the study of the kinetic curves of the process of longitudinal impregnation, depending on the tension of the fibrous filler, as well as prediction of the dimensions of the impregnating bath.

The above aspects are discussed in this article.

1.3. Research Methodology (Materials and Methods). The method of investigation is a complex one, which involves the 
use of set theory, theory of integral geometry and geometric probabilities, computer-aided design systems, system analysis, computational methods and computer graphics, experimental-statistical modeling, and microstructural analysis of thin sections of solidified composites with subsequent confirmation of the adequacy of the results obtained by experiments and tests.

The subject of the study is geometric and computer models of the basic technological process of "free" impregnation of oriented fibrous fillers (fiberglass and organofiber) as capillary-porous bodies with liquid polymeric binders in the form of liquid epoxy compositions. In the process of microstructural analysis, histograms of the distribution of distances between adjacent fibers in the cross section of the section were constructed. The obtained analytical dependencies of the distribution curves were used to model the structural parameters of oriented fibrous fillers as capillary-porous bodies, including porosity $\varepsilon$, effective capillary radius $r_{\mathrm{ef}}$, and specific surface area $S_{\mathrm{ss}}$. We studied the kinetic curves of the longitudinal impregnation process depending on the tension force of the fibrous filler, as well as the relationship of the structural parameters of the composite model and the kinetic parameters of the capillary impregnation process. The number of tests per each variable parameter under investigation was (7-10) with a reliability of $P=95 \%$.

\section{Results and Discussion}

2.1. An Analysis of Approaches to Modeling the Structure of the Cross Section of Thin Section of Oriented Fibrous Composites. According to the generally accepted concept, two basic approaches to the design of structural and technological parameters of technical molding tools and calculation of structural elements of composites based on OFFs are distinguished: phenomenological and structural [12]. Within the framework of the first approach, the composite is considered as a solid (consolidated) material with a certain tensor of reduced elastic (viscoelastic) characteristics. The second approach is based on the assumption of heterogeneity of the properties of the analyzed continuum, which contains reinforcing cylindrical inclusions (macro-fibers) of arbitrary cross section.

The first approach because of the simplicity of its prerequisites is more common. At the same time, it can only be regarded as an approximate one. This is due to the fact that the properties of constituent structural elements of composites (tensile strength, modulus of elasticity, etc.) on the basis of OFFs differ significantly between them (for example, by an order of magnitude or more). Therefore, the reduced (averaged) physicomechanical constants of such a monolithic system often lose physical meaning.

At the same time, the second approach allows us to more adequately model the parameters of the technology, as well as predict the stress-strain state of the structures taking into account the specific type of loading. This is due to the fact that the real structure of the studied composites is based on OFFs. So, for example, when modeling the parameters of the basic technological processes for molding reactoplastic
PCMs, in particular, the processes of free capillary impregnation and "wet" winding, new model physicomathematical and procedural-computerized approaches are used to describe the transfer of the impregnating liquid in the structure of OFFs. The latter are realized using geometric (structural) models [39].

At present it is primarily (1) structural-network models of porous and corpuscular media of various structures-"Pore Network Models" (random, regular, and other grids and regular packages); (2) methodology and mathematical apparatus of the theory of percolation (impregnation or leakage of liquids through porous materials); for these processes, it is the consideration of the transfer conditions of the impregnating fluid in the filler framework depending on the statistical characteristics of the gratings that model dispersed or porous media, the analogue of OFF (percolation in bonds, percolation in knots) [58,59]; (3) cluster analysis (cluster-bundle)-statistical identification of isolated structures [60]; in the transport problems (impregnation of OFFs with liquid polymeric binders), assemblages-clusters of conducting (or impregnated) regions, particles, molecules, etc., their dimensions, characteristics, and connection with the transportable properties of the liquid medium are studied; (4) fractal analysis-"Fractal Theory"-modeling and studying the properties of geometric elements (lines, surfaces, volumetric formations), taking into account the change in geometric values (length, area, volume) when the scale of measurement is changed [61].

In this case, often all of the above four approaches are used together. It should be noted that, in the simulation, the fibrous structure of capillary-porous bodies in the general case can not be regarded as a simple sum of linear capillaries with the same cross section. In practice, capillaries in fibrous systems differ significantly from cylindrical tubes [12]. In addition, as a rule, the capillary is not circular, and its walls are smooth. Considering the structure of OFF as a capillaryporous body, it should also be taken into account that the shape and area of the cross section of real capillaries can vary over a wide range along its length [62]. Also, capillaries can branch, join, and terminate in cul-de-sacs or large-volume pores [62].

In the general case, the concepts of capillary-porous media are much broader than those described above. Moreover, the more complex the structural model of such bodies, the more complex its geometric model and mathematical modeling of the processes of transport of a liquid medium in it. Therefore, it is necessary to find a certain balance when choosing an adequate geometric (structural) model of capillary-porous bodies for a particular technological process [1].

\subsection{Some Developed Approaches to Determining the $\mathrm{Pa}$ -} rameters of the Structural Model of Composites. The abovementioned data predetermines the need for more detailed consideration of the structural (physical, mathematical, and geometric) models of capillary-porous bodies, which are extremely diverse. At the same time, the common for them is the presence of a solid phase (OFF) and a system of voids in 
the form of channels and cavities. The solid phase of such bodies is called the skeleton of the body, and the system of voids is called the pore space. The volume fraction of voids is the porosity of $\varepsilon$, the surface of voids is the inner surface, and its value, referred to as unit volume of the body, is the specific surface area $S_{\mathrm{ss}}[39,42]$.

Some approaches to determining the parameters of the structural model of composites are shown in the example of designing parameters for the process of capillary impregnation of OFFs with liquid polymer binders. When determining the parameters of the kinetic equation of the "free" capillary impregnation process [63], it is necessary to correctly determine the structural characteristics of the OFFs on the basis of its adequate geometric model. As such characteristics, in the most general case, the porosity $\varepsilon$, the specific inner surface $S_{\text {ss }}$, and the effective (hydraulic) capillary radius $r_{\mathrm{ef}} \mathrm{OFF}$, are considered. Thus, four approaches to the determination of the parameters of the geometric model of the structure of OFF, including its effective (hydraulic) capillary radius $r_{\mathrm{e}}$, were developed [64-66].

The first of them (No. (1)) is based on the microstructural analysis of the cross section of an epoxy composite on the basis of OFF. The second (No. (2)) is the definition of $r_{\mathrm{ef}}$ on the basis of an analysis of the typical kinetic curve of the "free" impregnation process. The third (No. (3)) is the definition of $r_{\mathrm{ef}}$ on the calculated value for the model case of a highly compacted OFF of circular cross section (for hexagonal packing of fibers). The fourth (No. (4)) is the determination of the $r_{\mathrm{ef}}$ depending on the force of its tension $N$ during impregnation.

The latter approach is based on the assumption that, as a result of the tension of the fibrous filler, the $r_{\text {ef }}$ value will change, namely, decrease. In this case, the impregnation rate will decrease both as a result of a change in the structure and as a result of a decrease in the thickness of the OFF. Therefore, the processing of the experimental kinetic impregnation curves obtained for various values of the tension force $N$ of OFF allows one to obtain the desired experimental-statistical dependence $r_{\mathrm{ef}}=f(N)$.

Figure 7 shows the characteristic experimental dependence of the effective capillary radius of the oriented glass filler $r_{\mathrm{ef}}=f(N)$ upon its impregnation with epoxy composition (EC).

Obviously, according to the developed approach, for the studied impregnation temperatures $T$, it is also necessary to find the experimental dependencies $r_{\mathrm{ef}}=f(N)$, that is, to construct a family of these experimental curves. After that, using the methods of experimental-statistical modeling, it is necessary to find the coefficients of the approximating equation for a specific value of $N$, that is, to find the desired value of $r_{\mathrm{ef}}$.

So, for example, for the curve shown in Figure 7, a similar equation can be written as

$$
\begin{aligned}
r_{\mathrm{ef}}(N)= & -6.27 \cdot 10^{-7} \cdot X^{3}+0.000110617 \cdot X^{2} \\
& -0.007199145 \cdot X+0.32869,
\end{aligned}
$$

where $X$ denotes the specific tensile force $N(\mathrm{~N} / \mathrm{m})$ of the woven filler during the impregnation process.

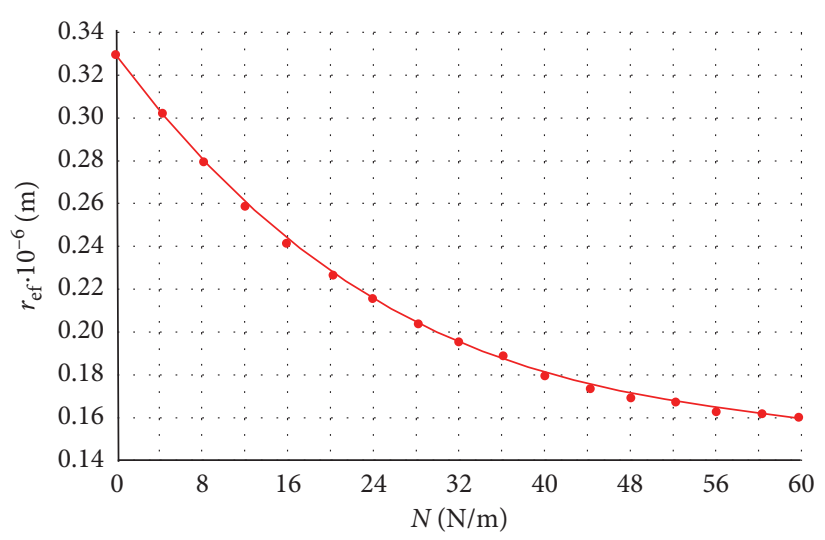

Figure 7: Dependence of the calculated value of $r_{\mathrm{ef}}$ oriented glass filler on the tensile force $N$ at an impregnation temperature of $T=50^{\circ} \mathrm{C}$.

The implementation of the last three above-mentioned approaches (No. 2-No. 4) is not particularly complicated. At the same time, the first of these approaches (No.1) deserves a separate consideration. This is due in particular to the fact that the first approach is applicable for determining the structural characteristics for both optically transparent and optically opaque (e.g., metal fiber) oriented composite fiber media.

This approach is based on an experimental study of the microstructure of such solidified media and the construction of the corresponding distribution curves characterizing the parameters of this microstructure. Figure 8 shows a characteristic photograph of the microsection of the unidirectional fibrous epoxy PCM.

First of all, an adequate model of the structure of OFF must take into account the stochastic character of the distribution of fibers in the structure of the real composite [64-66]. On the basis of the found parameters of the geometric model of the structure of OFF, optimum selection of the optimal reinforcement scheme and development of rational engineering at the design stage of the molded structure are carried out in the optimum. A prognosis is also made for the stress-strain state of the structure, taking into account the nature of the acting load [67]. A variant of the structure of OFF is a geometric model of the structure of a capillary-porous body, which consists of a system of paralleltortuous capillaries of different radii. It can be formalized with the help of a pore distribution function of radius $\rho$ with respect to the dimensions of $\varphi(\rho)$ [63].

The developed approach to the determination of the effective capillary radius of the $r_{\mathrm{ef}}$ is as follows. The microsection of the oriented PCM is made in a direction transverse to the fibers. Further, the length distribution of the ext-chords of the fibers or the distribution function $\varphi$ $(\rho)=G(\ell)$ of pores by size $\ell$ is experimentally investigated. In this case, the "ext-chords" denote the distances between adjacent fibers in the cross section of the composite (i.e., equivalent diameter, or twice the radius of the pore ref). In the general case, the lengths of the ext-chords are random.

When the condition of the best approximation of the theoretical and experimental distribution curves is satisfied, 
the porosity $\varepsilon$, the specific inner surface $S_{\mathrm{ss}}$, and the effective (equivalent) capillary radius ref of the desired OFF as a capillary-porous body are computed using the ext-chords length distribution function $\varphi(\rho)=G(\ell)$.

2.3. An Analytical Determination of the Structural Characteristics of Oriented Fibrous Fillers. The porosity $\varepsilon$ in a capillary-porous body that simulates the OFF structure can be defined as $[42,59]$

$$
\varepsilon=N^{0} \pi \beta_{i} \int_{0}^{\infty} \rho^{2} \varphi(\rho) \mathrm{d} \rho=\frac{N^{0} \pi \beta_{0} \bar{\rho}^{2}}{4}=\frac{N^{0} \pi \beta_{0} \bar{\rho}^{2}}{4} \int_{0}^{\infty} \rho^{2} \varphi(\rho) \mathrm{d} \rho,
$$

$E=m c^{2}$ where $N^{0}$ is the number of measurements between the fibers in the plane of thin section oriented PCM.

In turn, the specific internal surface $S_{\mathrm{ss}}$ of such an OFF can be defined as

$$
S_{\mathrm{ss}}=k_{0} N^{0} \pi \beta \int_{0}^{\infty} \rho \varphi(\rho) \mathrm{d} \rho=k_{0} N^{0} \pi \beta \bar{\rho},
$$

where the coefficients $k_{0}$ and $\beta_{0}$ can be interpreted as correction factors that take into account the specificity of the OFF structure as a capillary-porous medium, respectively, the surface roughness and tortuosity of the equivalent cylindrical capillary [62].

If we take as the effective (or equivalent) capillary radius $r_{\mathrm{ef}}$ of the sought OFF hydraulic radius, which is determined by analogy with a cylindrical capillary as the ratio of twice the pore volume to their surface, then we will have

$$
r_{\mathrm{ef}}=\frac{2 \varepsilon}{S_{\mathrm{ss}}}=\frac{2}{k_{0}}\left[\frac{\int_{0}^{\infty} \rho^{2} \varphi(\rho) \mathrm{d} \rho}{\int_{0}^{\infty} \rho \varphi(\rho) \mathrm{d} \rho}\right] .
$$

Typically, the experimental curve for the distribution of extinction lengths of the OFF's ext-chords, obtained on the basis of $N^{0}=2500$ measurements in the plane of the section, is shown in Figure 9.

For practical calculations, the model function of the distribution of the length of the ext-chords of fibers, indicated in Figure 9 as $(-)$, which minimizes deviations from experimental ordinates $(O)$, is convenient to be described in this form:

$$
G(\ell)=\varphi(\rho)=\frac{c_{k} \cdot b^{\left(1 / c_{k}\right)}}{\Gamma\left(1 / c_{k}\right)} e^{-\left(b_{k} \cdot \rho^{c_{k}}\right)},
$$

where $b_{k}, c_{k}$ are positive constants determined from the condition of the best approximation of the ordinates of the theoretical curve (5) to the experimental ordinates; $\Gamma\left(1 / c_{k}\right)$ is Euler gamma-function.

For case (5), the mathematical expectation of a random variable $\rho$ is

$$
M(\rho)=\int_{0}^{\infty} \rho \varphi(\rho) \mathrm{d} \rho=\frac{\Gamma\left(2 / c_{k}\right)}{\Gamma\left(1 / c_{k}\right) \cdot b^{\left(1 / c_{k}\right)}} .
$$

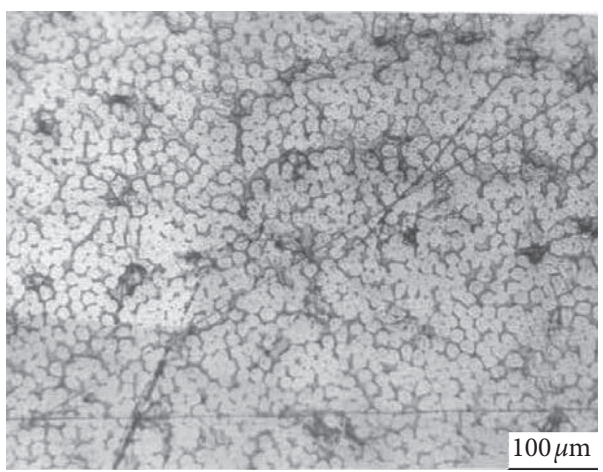

FIGURE 8: A characteristic photograph of the microsection of the unidirectional fibrous PCM made from oriented epoxy organoplastic. Magnification 1200.

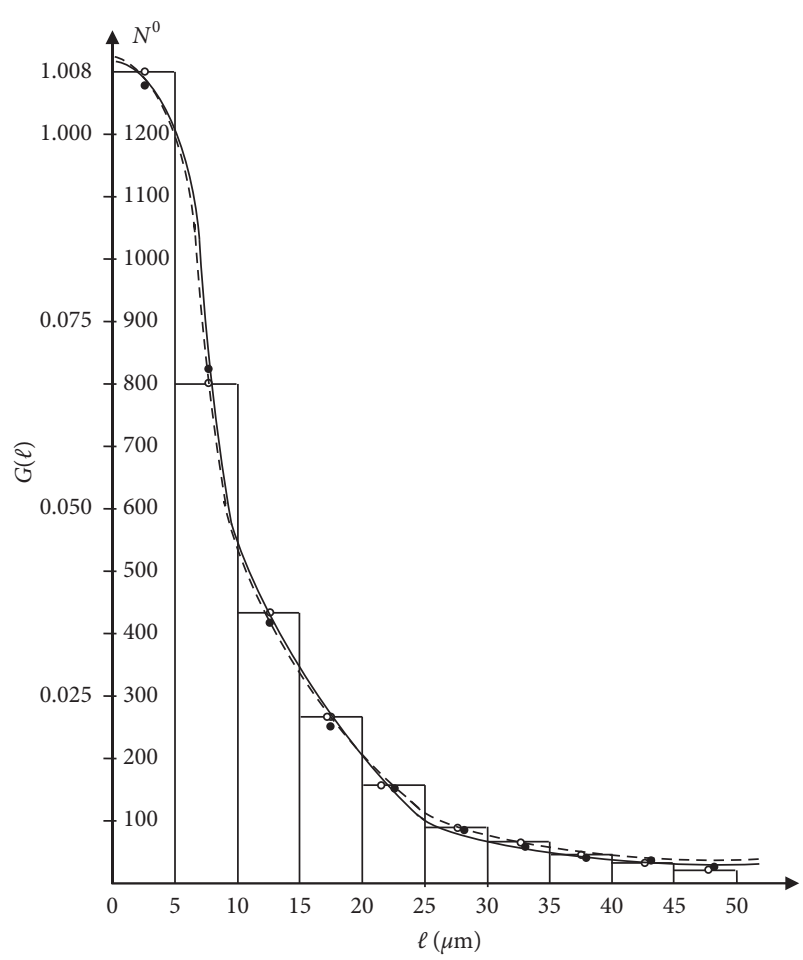

Figure 9: The histogram and the curve of the distribution of the lengths of the ext-chords of adjacent fibers (circles) $G(\ell)$ : experimental ordinates $(\bigcirc)$; model ordinates $(\bullet)$ and a model curve for the distribution of the lengths of the ext-chords of neighboring fibers (-); $N^{o}$ is the number of measurements.

The formula for porosity will take the following form:

$$
\varepsilon=\frac{N^{0} \pi \beta_{0}}{4} \int_{0}^{\infty} \rho^{2} \varphi(\rho) \mathrm{d} \rho=\frac{N^{0} \pi \beta_{0}}{4} \frac{\Gamma\left(3 / c_{k}\right)}{\Gamma\left(1 / c_{k}\right) \cdot b^{\left(2 / c_{k}\right)}},
$$

and formula (4) for equivalent capillary radius $r_{\mathrm{ef}}$ is written as

$$
r_{\mathrm{ef}}=\frac{2 \varepsilon}{S_{\mathrm{ss}}}=\frac{2}{k_{0}}\left[\frac{\int_{0}^{\infty} \rho^{2} \varphi(\rho) \mathrm{d} \rho}{\int_{0}^{\infty} \rho \varphi(\rho) \mathrm{d} \rho}\right]=\sqrt{2} \frac{\Gamma\left(1 / c_{k}\right)}{\Gamma\left(2 / c_{k}\right) \cdot b^{\left(1 / c_{k}\right)}} .
$$


So, with the number of measurements of the distance between the fibers $N^{0}=2500$ in the plane of the cut-off OFF (see Figures 8 and 9) and with the values $\beta_{0}=1.1, \mathrm{~K}_{\mathrm{O}} \approx \sqrt{2}$ according to [40], the mathematical expectation (6) is $M$ $(\rho)=8.158 \mu \mathrm{m}$.

In this case, in equation (4), the constants $b_{k}=0.129$, $c_{k}=0.9812$, and by dependency (7), the porosity is $\varepsilon=0.28$ (i.e., the reinforcement ratio or volume content OFF in the PCM structure is $1-\varepsilon=0.72$ ), and by equation (8), the effective (equivalent) capillary radius $r_{\mathrm{ef}}=5.96 \mu \mathrm{m} \approx 6 \mu \mathrm{m}$.

2.4. Designing the Parameters of Technology and Equipment for the Production of Composites. The tasks of designing technology and equipment for the production of traditional and constructional PCMs are aimed at identifying and studying the interrelations between the structural, mechanical, and geometric parameters of products, on one hand, and the technological factors of their production, on the other.

Thus, for example, the adequate geometric model of the structure of OFFs is used to determine the parameters of the refined mathematical model of the technological process of "free" impregnation of OFF with ECs. This is done on the basis of using the classical theory of filtration for laminar flow of a viscous incompressible non-Newtonian fluid in a capillary-porous body, using approaches in studies $[42,59,62]$.

2.5. The Method of Modeling the Parameters of the Process of "Free" Impregnation of Oriented Fibrous Fillers with Liquid Polymer Binders. The kinetic equation of the capillary impregnation process allows predicting the design and technological parameters of the process. For example, to find the dimensions of the impregnation bath (length and height), it is desirable to estimate the maximum height and speed of the process of longitudinal "free" impregnation of OFF with liquid $\mathrm{EC}$ at a given impregnation temperature.

Wetting ability $\sigma \cos \theta$, calculated by the maximum height of the EC rise along the fiber under the action of surface tension forces, can be found by the formula $[42,59]$

$$
\sigma \cos \theta=\frac{h_{\infty} \cdot \gamma g R_{k}}{2} \Rightarrow h_{\infty}=\frac{2 \sigma \cos \theta}{\gamma g R_{k}},
$$

where $h_{\infty}$ is the maximum lifting height (longitudinal impregnation) of the polymer liquid; $R_{k}$ is the radius of the capillary.

The current time of longitudinal "free" impregnation $t$ and time to reach saturation $t_{\infty}$ of OFF with liquid EC, according to [63], is written as follows:

$$
t=\frac{h^{2} \eta}{2 r_{\mathrm{ef}} \sigma \cos \theta} \Rightarrow t_{\mathrm{\infty}}=\frac{h_{\mathrm{\infty}}^{2} \eta}{2 r_{\mathrm{ef}} \sigma \cos \theta} .
$$

By differentiating equation (10), one can find the impregnation rate $v_{\mathrm{imp}}$ (until the impregnation saturation point is OFF):

$$
v_{\text {imp }}=\frac{\mathrm{d} h}{\mathrm{~d} t}=\frac{r_{\mathrm{ef}} \sigma \cos \theta}{4 h \eta} .
$$

For curve 3 (tension values $N=0.3 \mathrm{~N} / \mathrm{m}$ ), we give the values of equations (9)-(11) for the model distribution function of the lengths ext-chord of fibers, which minimizes the deviations from the experimental ordinates $(O)$-see Figure 10.

The dynamic viscosity of the EC solution at this temperature of $T=40^{\circ} \mathrm{C}$ is $0.48 \mathrm{~Pa} \cdot \mathrm{s}$, and the wetting ability is $\sigma \cos \theta=11.9 \cdot 10^{-2} \mathrm{~N} / \mathrm{m}$. In particular, for the experimental kinetic curve of "free" impregnation 3 (Figure 10), the parameters turned out to be $h_{\infty}=3.15 \cdot 10^{-3} \mathrm{~m}$; $t_{\infty}=10.5 \mathrm{~s} ; v_{\text {imp }}=1.03 \cdot 10^{-3} \mathrm{~m} / \mathrm{s}$.

Analytically obtained kinetic equations of "free" longitudinal impregnation of oriented and woven fibrous fillers with liquid ECs allow to predict the impregnation time and the speed of broaching the fibrous filler through the impregnating bath, and also to design its dimensions depending on the amount of tension force impregnation.

It is convenient to use adequate structural models for numerical modeling of the OFF parameters, as well as for predicting the parameters of the capillary impregnation process. For extremely reinforced PCMs based on OFF, a geometric model of Vanin's medium is used [67]. Elementary cells of this geometric model are shown in Figure 11.

The geometric model of Vanin's medium (Figure 11) is represented by a system of circular cylindrical fibers whose axes are parallel and located at the nodes of the unit cell (a doubly periodic lattice of parallelograms). The space between the fibers is filled with a binder polymer medium, and the fiber cross-sections must not overlap [1]. And the plane of the section of the investigated composite on the basis of the OFF is modeled by a doubly periodic continuation of the unit cell.

As the packing parameters of cylindrical fibers (Figure 11), the lengths of its sides $\omega_{1}$ and $\omega_{2}$ and the angle between them $\alpha$ are chosen. The unit of measurement of distances in the model plane is, for convenience, the base length of the side of the unit cell $\omega_{1}$. The criterion for the adequacy of the structural model of the composite under study is the coincidence with a certain degree of accuracy (at least $90 \%$ of the component) of the coefficients and probabilistic characteristics of the curves of the distributions of the lengths of the chords of circles and the distances between neighboring fibers (ext-chords). Also, in the specified confidence limits, the values of the "porosity" $\varepsilon$ and the relative content of the binder in the model plane and in the natural composite on the basis of OFF, respectively, should be found.

These quantities are determined as a result of measurements carried out along a random secant line. The latter "rushes" randomly in the model plane and in the plane of microsections of the cross section of the full-scale shell structure. Subsequently, knowing the parameters of an adequate structural model (see Figure 11), one can predict the stress-strain state of a real PCM based on the corresponding analytical dependencies [1]. 


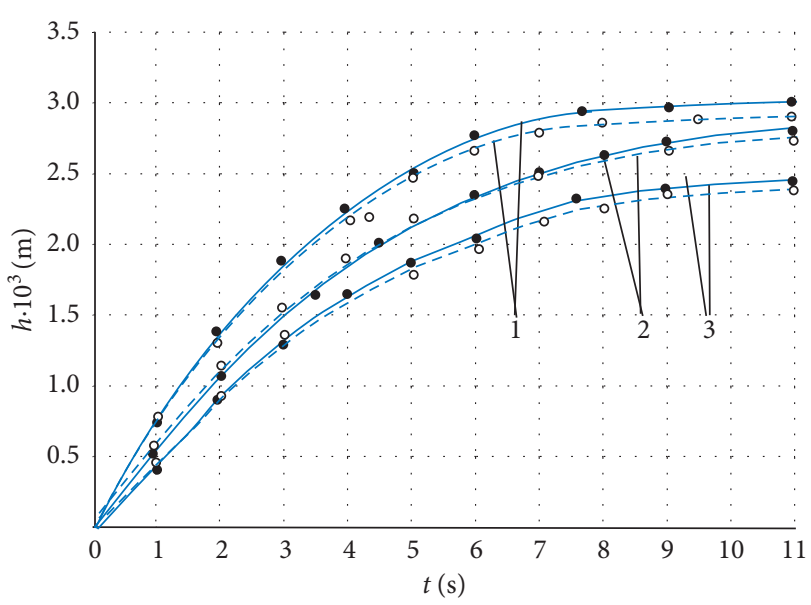

FIGURE 10: The characteristic kinetic curves of the longitudinal "free" impregnation of the organic fiber bundle of the SVM by EC brand ED-20 at a temperature of $40^{\circ} \mathrm{C}$ and with variable tension forces $N_{t}$ per OFF thread (tension values $N_{t}$ are shown in curves): $1-N=0.05 \mathrm{~N} / \mathrm{m} ; 2-N=0.20 \mathrm{~N} / \mathrm{m} ; 3-N=0.3 \mathrm{~N} / \mathrm{m} ;(\bullet)$-theoretical curve constructed by equation (9); (O)-experimental values; $t$-time.

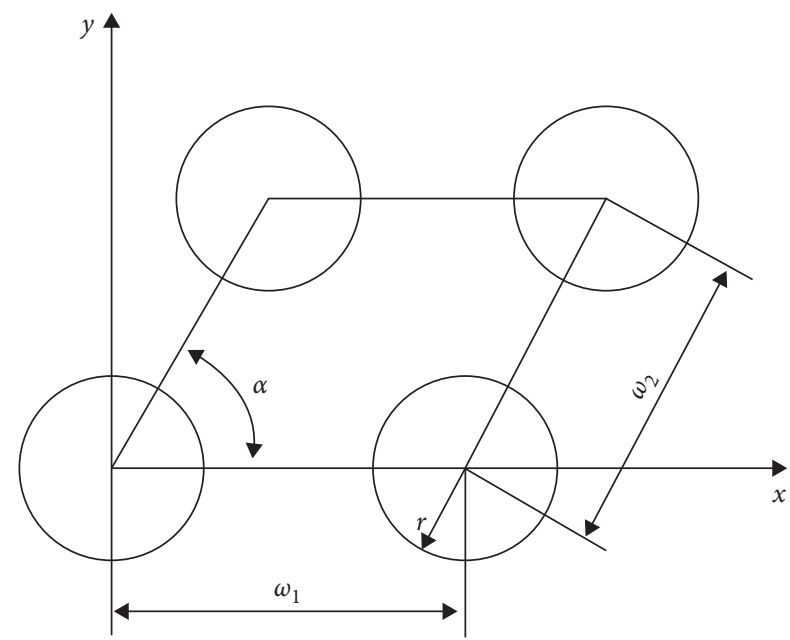

FIGURE 11: The geometric Vanin's model for extremely reinforced PCMs medium.

The method of determining the parameters of an adequate geometric model of the structure of an OFF (see Figure 11) is as follows (see Figures 12(a)-12(d)) [32].

From the model of the structure of OFF, which in the general case consists of a system of parallel-winding capillaries of different radii (Figure 12(a)), and which is determined by the function of the distribution of pores of radius $\rho$ in terms of the sizes of $\varphi(\rho)$, they are transformed using prognostic analytical relations, for example, given in [63], to averaging the structure of OFF as a regular structure of linear capillaries with the same longitudinal section (Figure 12(b)).

The scheme for filling the impregnating liquid of the latter is shown in Figures 12(c) and 12(d) (cross section of the OFF). Those, as a result, pass to the impregnation scheme for a single capillary of length $\ell_{c}$ (Figure 12(d)), whose diameter is $2 r_{\text {ef. }}$. It should be noted that the above-described approach to the construction of model distribution curves (obtained analytically and as a result of numerical experimental) of chord lengths and extensions in the structure of OFF was adopted from the theory of integral geometry and geometric probabilities $[68,69]$ and adapted to the OFF in the studies [32].

Within the framework of this theory, we study geometric objects of a random character (point fields, lines, mosaics, etc.) that are invariant under the transformation groups of space. In particular, convex figures are studied in the theory of geometric probabilities, which are intersected by random lines. And these figures can have a general shape, either convex or circular. The main method used involves placing a random line of length $L$, the so-called "secant," into the study area. After that, intersections with figures that do not have common points are measured.

Various questions of the geometric modeling of the structure of oriented macro-fiber reactoplastic composites used to predict the design and technological parameters of thermosetting molding, as well as to calculate their stressstrain state, can be found in $[7,12,67]$. In particular, the influence of structural parameters (reinforcement schemes) and technological parameters (properties of reinforcement and polymer matrix) on characteristics (operational properties) of hardened PCMs on their basis is considered. We also note that, in the geometric modeling of such structures, as well as the nature of the applied forces and the stresses arising in this process, depending on the stress-strain state, the use of symmetry principles is an effective method [7].

It should also be noted that, in order to find the dimensions of the impregnation bath (length and height), it is necessary to estimate the maximum height and speed of longitudinal "free" impregnation of OFFs with liquid ECs [70] at a given impregnation temperature according to the analytical dependencies briefly presented in [63]. To design the optimal tension force of impregnated fibrous fillers in circumferential "wet" winding allows the study of experimental results on the influence of technological impregnation regimes under a certain tension force on the strength of impregnated and cured fibrous fillers [71].

Moreover, in order to minimize material and time costs, as a rule, a technique of structural and parametric modeling of the design and technological parameters of technology and equipment (tools) for the production of reactoplastic oriented reinforced PCMs taking into account the structural organization of OFFs is used [72].

Also, based on the developed approach, PCMs modeling is possible using both chemical modification and combined physicochemical modification of the components of PCMs including OFFs of rope and woven types [73, 74]. At the same time, the modification should ultimately lead to improved PCMs operational characteristics [75] and also contribute to increasing the productivity of molding and energy saving when receiving reinforced composites due to the ordering of their structure, improvement of interfacial interaction at the "fiber-polymer matrix" interface, and reduction of voids in the formed composite [76]. 


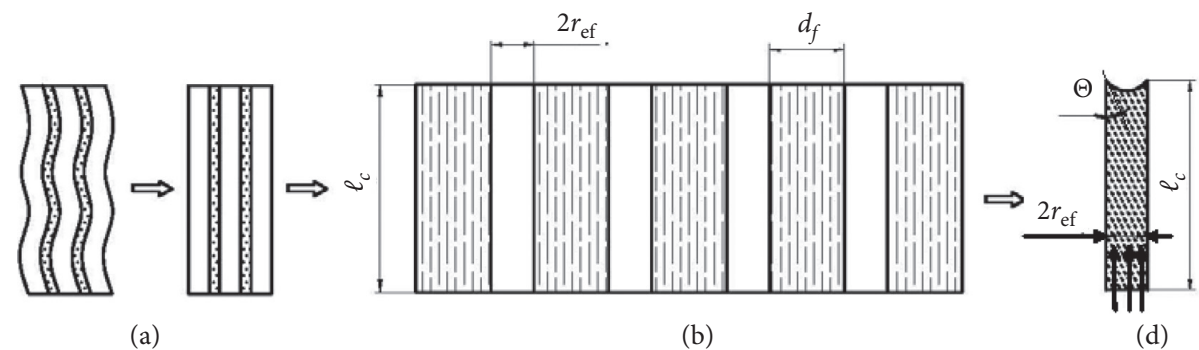

(a)

(b)

(d)

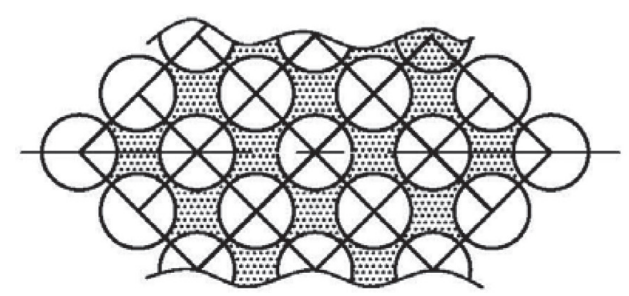

(c)

FIGURE 12: An illustration of the implementation of the methodology for constructing (a)-(d) an adequate geometric model of the microstructure of OFF in the technology of their "free" impregnation with a liquid epoxy binder: $r_{\mathrm{ef}}$ is the effective capillary radius; $d_{f}$ is the average fiber diameter; $\ell_{c}$ is capillary length; $\theta$ is the wetting contact angle.

\section{Conclusions}

(1) Features of modeling the structure of oriented macrofiber reactoplastic composites using the phenomenological and structural approaches are analyzed. The technique used to determine the structural characteristics of oriented macrofibrous fillers as capillary-porous bodies is described on the basis of the use of an adequate geometric model of the structure. The parameters of the structural model are determined using the apparatus of the theory of geometric probabilities and integral geometry.

(2) The porosity, specific internal surface, and effective (hydraulic) capillary radius of oriented macrofibrous fillers are considered as the main structural characteristics of the capillary-porous body. The latter simulates the real interfiber space of a reinforcing fibrous filler in the process of its capillary impregnation by ECs.

(3) Analytically obtained kinetic equations of "free" longitudinal impregnation of oriented and woven fibrous fillers with liquid ECs allow to predict the impregnation time and the speed of broaching the fibrous filler through the impregnating bath, and also to design its dimensions depending on the amount of tension force impregnation.

(4) The use of the described approach provides the fundamental possibility of determining the typical structural models of existing and new oriented composites.

\section{Data Availability}

The datasets generated and/or analysed during the current study are available from the corresponding author on reasonable request.

\section{Conflicts of Interest}

The authors declare that there are no conflicts of interest regarding the publication of this paper.

\section{References}

[1] K. K. Kar, Composite Materials. Processing, Applications, Characterizations, Springer-Verlag, Berlin, Germany, 2017.

[2] R. E. Shalin, Polymer Matrix Composites, Springer Science \& Business Media, Berlin, Germany, 2012.

[3] B. G. Falzon and R. S. Pierce, "Thermosetting composite materials in aerostructures," in Revolutionizing Aircraft Materials and Processes, S. Pantelakis and K. Tserpes, Eds., Springer, Cham, Switzerland, 2020.

[4] C. Soutis, "Fibre reinforced composites in aircraft construction," Progress in Aerospace Sciences, vol. 41, no. 2, pp. 143-151, 2005.

[5] B. Esp, Practical Analysis of Aircraft Composites, Grand Oak Publishing, Atlanta, GA, USA, 1st edition, 2017.

[6] G. G. Timothy, Advanced Composites Manufacture, Wiley, New York, NY, USA, 1997.

[7] V. V. Vasiliev and E. V. Morozov, Advanced Mechanics of Composite Materials and Structures, Elsevier, Amsterdam, Netherlands, 4th edition, 2018.

[8] V. A. Nelyub, "The use of polymer composite materials in shipbuilding for the repair of ship superstructures," Remont. Vosstanovlenie. Modernizatsiya, vol. 5, pp. 21-24, 2013, in Russian.

[9] Z. Benzait and L. Trabzon, "A review of recent research on materials used in polymer-matrix composites for body armor application," Journal of Composite Materials, vol. 52, no. 23, pp. 3241-3263, 2018.

[10] V. Grumezescu and A. Grumezescu, Materials for Biomedical Engineering: Thermoset and Thermoplastic Polymers, Elsevier, Amsterdam, Netherlands, 2019.

[11] F. T. Wallenberger and P. A. Bingham, Fiberglass and Glass Technology. Energy-Friendly Compositions and Applications, Springer US, New York City, NY, USA, 2010. 
[12] X.-S. Yi, D. Shanyi, and Z. Litong, "Composite materials engineering," Fundamentals of Composite Materials, vol. 1, Springer, Singapore, 2018.

[13] X.-S. Yi, D. Shanyi, and Z. Litong, "Composite materials engineering," Different Types of Composite Materials, vol. 2, Springer, Singapore, 2018.

[14] P. K. Mallick, Composite Engineering Handbook, Marcel Dekker, New York, NY, USA, 1997.

[15] M. Biron, Thermosets and Composites: Material Selection, Applications, Manufacturing and Cost Analysis, William Andrew, Norwich, CT, USA, 2014.

[16] A. R. Bunsell and J. Renard, Fundamentals of Fibre Reinforced Composite Materials, CRC Press, Boca Raton, FL, USA, 1st edition, 2005.

[17] D. D. L. Chung, Composite Materials Science and Applications, Springer, London, UK, 2nd edition, 2010.

[18] R. E. Shalin, "Polymer matrix composites," in Series Soviet Advanced Composites Technology, vol. 4Dordrecht, Netherlands, Springer, 1995.

[19] N. Yang, "Advanced polymer matrix composites," in Composite Materials Engineering, X. S. Yi, S. Du, and L. Zhang, Eds., vol. 2Singapore, Springer, 2018.

[20] J.-M. Berthelot, Classical Laminate Theory, Springer, New York, NY, USA, 1999.

[21] M. Bruyneel, Optimization of Laminated Composite Structures: Problems, Solution Procedures and Applications, Nova Science Publishers, Composite Materials Research Progress, London, UK, 2008.

[22] R. Khandan, S. Noroozi, P. Sewell, and J. Vinney, "The development of laminated composite plate theories: a review," Journal of Materials Science, vol. 47, no. 16, pp. 5901-5910, 2012.

[23] Y. Xu, J. Zhu, Z. Wu, Y. Cao, Y. Zhao, and W. Zhang, "A review on the design of laminated composite structures: constant and variable stiffness design and topology optimization," Advanced Composites and Hybrid Materials, vol. 1, no. 3, pp. 460-477, 2018.

[24] A. V. Gondlyakh, A. O. Chemeris, A. E. Kolosov, A. L. Sokolskiy, and S. I. Antonyuk, "Simulation of delamination processes of multilayer mechanical structures in mechanical engineering," in Proceedings of the 2nd Grabchenko's International Conference on Advanced Manufacturing Processes (InterPartner-2020), V. Ivanov, Ed., Springer, Odessa, Ukraine, Lecture Notes in Mechanical Engineering, Odessa, Ukraine, September 2020.

[25] D. Gay, S. V. Hoa, and S. V. Tsai, Composite Materials: Design and Applications, CRC Press LLC, Boca Raton, FL, USA, 4th edition, 2003.

[26] D. Gay, Composite Materials: Design and Applications, CRC Press, Boca Raton, FL, USA, 3rd edition, 2014.

[27] A. B. Strong, Fundamentals of Composites Manufacturing: Materials, Methods and Applications, Society of Manufacturing Engineers, Dearborn, MI, USA, 2008.

[28] K. K. Chawla, Composite Materials. Science and Engineering, Springer-Verlag, New York, NY, USA, 2012.

[29] N. S. Muralisrinivasan, "Basics of troubleshooting in plastics processing: an introductory practical guide," in Handbook of Plastics Testing and Failure Analysis, p. 240, 3rd edition, Wiley, Hoboken, NJ, USA, 2011.

[30] A. G. Bratukhin and V. S. Bogolyubov, Composite Manufacturing Technology, Springer Science \& Business Media, Berlin, Germany, 2012.

[31] A. E. Kolosov and I. A. Repelis, "Saturation of fibrous fillers with polymer binders 5 . Optimization of parameters of the winding conditions," Mechanics of Composite Materials, vol. 25, no. 3, pp. 407-415, 1989.

[32] A. E. Kolosov, G. A. Virchenko, E. P. Kolosova, and G. I. Virchenko, "Structural and technological design of ways for preparing reactoplastic composite fiber materials based on structural parametric modeling," Chemical and Petroleum Engineering, vol. 51, no. 7-8, pp. 493-500, 2015.

[33] D. É. Sidorov, A. E. Kolosov, O. V. Pogorelyi, and A. A. Gur'eva, "Engineering approach to the determination of the radiation field of a polyethyleneterephthalate (PET) medium under radiant heating," Journal of Engineering Physics and Thermophysics, vol. 88, no. 6, pp. 1409-1415, 2015.

[34] A. E. Kolosov, G. A. Virchenko, E. P. Kolosova, and S. L. Shambina, "Simulation of the parameters of an ultrasonic dosed cavitator with radiative plate. Part 1. Analysis of effective technical means of ultrasonic exposure and a technique of variant structural-parametric design," Chemical and Petroleum Engineering, vol. 54, no. 7-8, pp. 531-541, 2018.

[35] G. A. Virchenko, A. E. Kolosov, E. P. Kolosova, and S. G. Virchenko, "Simulation of the parameters of ultrasonic dosing device with radiative plate. Part 2. Optimization of design and technological parameters," Chemical and Petroleum Engineering, vol. 54, no. 7-8, pp. 605-612, 2018.

[36] V. V. Vanin, G. A. Virchenko, A. E. Kolosov, and E. P. Kolosova, "Simulation of the parameters of ultrasonic dosing cavitation device with radiative plate. Part 3. Computer-aided variant 3D design," Chemical and Petroleum Engineering, vol. 54, no. 9-10, pp. 694-700, 2019.

[37] N. B. Uriev, Technology of Dispersed Systems and Materials: Physicochemical Dynamics of Structure Formation and Rheology, John Wiley \& Sons, Hoboken, NJ, USA, 2016.

[38] Yu.M. Tarnopolsky, I. G. Zhigun, and V. A. Polyakov, Spatially Reinforced Composite Materials, Catalog, Mashynostroyenie, Moscow, Russia, in Russian, 1987.

[39] V. I. Konovalov, T. Kudra, and N. Ts. Gatapova, "Modern issues of transport theory in drying," Bulletin of Tambov State Technical University, vol. 14, no. 3, pp. 538-559, 2008.

[40] G. A. Akselrud and M. A. Altshuler, Introduction to CapillaryChemical Technology, Khimiya, Moscow, Russia, in Russian, 1983.

[41] L. I. Kheifets and A. V. Neymark, Mnogofaznye Protsessy V Poristykh Sredakh [Multiphase Processes in Porous Media, Khimiya, Moscow, Russia, in Russian, 1982.

[42] G. B. Shalun and E. M. Surzhenko, Layered Plastics, Khimiya, Leningrad, Russia, in Russian, 1978.

[43] H. M. El-Dessouky and M. N. Saleh, "3D woven composites: from weaviing tto manufacturing," in Recent Developments in the Field of Carbon Fibers, R. Khanna and R. Cayumil, Eds., InTechOpen, London, UK, pp. 51-66, 2018.

[44] G. Gardiner, Albany Engineered Composites: Weaving the Future in 3-D, CompositesWorld, Cincinnati, OH, USA, 2014, https://www.compositesworld.com/articles/albanyengineered-composites-weaving-the-future-in-3-d.

[45] H. Stiller, "Material intensity of advanced composite materials: results of asudy for the verbundwerkstofflabor bremen e.V." Wuppertal Papers, No. 90, Wuppertal Institute for Climate, Environment and Energy, Wuppertal, Germany, 1999, https://www.econstor.eu/handle/10419/49096.

[46] M. N. Saleh and C. Soutis, "Recent advancements in mechanical characterisation of $3 \mathrm{D}$ woven composites," Mechanics of Advanced Materials and Modern Processes, vol. 3, no. 1, p. 12, 2017.

[47] M. N. Saleh, Y. Wang, A. Yudhanto et al., "Investigating the potential of using off-axis $3 \mathrm{D}$ woven composites in composite 
joints' applications," Applied Composite Materials, vol. 24, no. 2, pp. 377-396, 2016.

[48] M. N. Saleh, A. Yudhanto, P. Potluri, G. Lubineau, and C. Soutis, "Characterising the loading direction sensitivity of 3D woven composites: effect of z-binder architecture," Composites Part A: Applied Science and Manufacturing, vol. 90 , pp. 577-588, 2016.

[49] C. McHugh, "Creating 3-D, one piece, woven carbon preforms using conventional weaving and shedding," SAMPE Conference Proceeding, vol. 45, no. 6, pp. 33-41, 2009.

[50] C. McHugh, "The manufacture of one piece woven three dimensional carbon fiber nodal structures," in Proceedings of the SAMPE Conference, Seattle, WA, USA, May 2010.

[51] X. Chen, X. Chen, L. W. Taylor, and L. Tsai, "An overview on fabrication of three- dimensional woven textile preforms for composites," Textile Research Journal, vol. 81, no. 9, pp. 932-944, 2011.

[52] C. Redman, H. Bayraktar, and M. Mcclain, "Curved beam test behavior of 3D woven composites," in Proceedings of the SAMPE, Seattle, WA, USA, 2014.

[53] H. Bayraktar, D. Ehrlich, J. Goering, and M. Mcclain, "3D woven composites for energy absorbing," in Proceedings of the 20th International Conference on Composite Materials, Copenhagen, Denmark, July 2015.

[54] H. M. EL-Dessouky, A. Snape, H. Tew et al., "Design, weaving and manufacture of a large 3D composite structure for automotive applications," in Proceedings of the 7th World Conference 3D Fabrics and their Applications, Roubaix, France, September 2016.

[55] H. M. EL-Dessouky, A. Snape, J. L. Turner et al., “3D weaving for advanced composite manufacturing: from research to reality," in Proceedings of the SAMPE Conference, Seattle, WA, USA, 2017.

[56] J. G. Hemrick, E. Lara-Curzio, E. R. Loveland, K. W. Sharp, and R. Schartow, "Woven graphite fiber structures for use in ultra-light weight heat exchangers," Carbon, vol. 49, no. 14, pp. 4820-4829, 2011.

[57] J. Jewell, R. Kennedy, and A. Menard, "Full-scale LEAP fan blade-out rig test yields outstanding results; advanced LEAP fan endurance test complete," in CFM Power Flight, CFM International LEAP, Columbus, OH, USA, 2011.

[58] M. J. Blunt, Multiphase Flow in Permeable Media: A Pore-Scale Perspective, Cambridge University Press, Cambridge, UK, 2017.

[59] T. Dang-Vu and J. Hupka, "Characterization of porous materials by capillary rise method," Physicochemical Problems of Mineral Processing, vol. 39, pp. 47-65, 2005.

[60] B. S. Everitt, S. Landau, M. Leese, and D. Stahl, Cluster Analysis, John Wiley \& Sons, Hoboken, NJ, USA, 5th edition, 2011.

[61] K. J. Falconer, Fractals: A Very Short Introduction, OUP Oxford, Oxford, UK, 2013.

[62] A. V. Luikov, Heat and Mass Transfer in Capillary-Porous Bodies, Elsevier, Amsterdam, Netherlands, 2014.

[63] A. E. Kolosov, "Impregnation of fibrous fillers with polymer binders. 1. Kinetic equations of longitudinal and transverse impregnation," Mechanics of Composite Materials, vol. 23, no. 5, pp. 625-633, 1988.

[64] A. F. Kichigin, A. E. Kolosov, V. V. Klyavlin, and V. G. Sidyachenko, "Probabilistic-geometric model of structurally inhomogeneous materials," Soviet Mining Science, vol. 24, no. 2, pp. 87-94, 1988.

[65] A. E. Kolosov and V. V. Klyavlin, "Determination of the parameters of a geometric model of the structure of directionally reinforced fiber composites," Mechanics of Composite Materials, vol. 23, no. 6, pp. 699-706, 1988.

[66] A. E. Kolosov and V. V. Klyavlin, "Several aspects of determination of the adequate model of the structure of oriented fiber-reinforced composites," Mechanics of Composite Materials, vol. 24, no. 6, pp. 751-757, 1989.

[67] G. A. Vanin, Micromechanics of Composite Materials, Naukova Dumka, Kiev, Ukraine, in Russian, 1985.

[68] R. Langevin, Integral Geometry from Buffon to Geometers of Today, Société Mathématique de France, Paris, France, 2015.

[69] R. Schneider and W. Weil, Stochastic and Integral Geometry, Springer Science \& Business Media, Berlin, Germany, 2008.

[70] J.-P. Pascault and R. J. J. Williams, Epoxy Polymers: New Materials and Innovations, Wiley VCH, Weinheim, Germany, 2009.

[71] A. E. Kolosov, I. A. Repelis, V. G. Khozin, and V. V. Klyavlin, "Impregnation of fibrous fillers with polymer binders. 2. Effect of the impregnation regimes on the strength of the impregnated fillers," Mechanics of Composite Materials, vol. 24, no. 3, pp. 373-380, 1988.

[72] P. K. Mallick, Processing of Polymer Matrix Composites, CRC Press, Boca Raton, FL, USA, 1st edition, 2017.

[73] A. E. Kolosov, V. I. Sivetskii, E. P. Kolosova et al., "Creation of structural polymer composite materials for functional application using physicochemical modification," Advances in Polymer Technology, vol. 2019, Article ID 3501456, 12 pages, 2019.

[74] A. E. Kolosov, V. I. Sivetskii, E. P. Kolosova et al., "Use of physico-chemical modification methods for producing of traditional and nanomodified polymeric composites with improved operational properties (a review)," International Journal of Polymer Science, vol. 2019, Article ID 1258727, 18 pages, 2019.

[75] S. L. Roginskii, M. Z. Kanovich, and M. A. Koltunov, HighStrength Glass Reinforced Plastics, Khimiya, Moscow, Russia, in Russian, 1979.

[76] A. E. Kolosov, A. V. Gondlyakh, E. P. Kolosova, D. E. Sidorov, and A. Khan, "Energy-efficient technology for producing epoxy polymers by ultrasonic treatment," in Proceedings of the 2nd Grabchenko's International Conference on Advanced Manufacturing Processes (InterPartner-2020), V. Ivanov, Ed., , Springer, Odessa, Ukraine, Lecture Notes in Mechanical Engineering, Odessa, Ukraine, September 2020. 